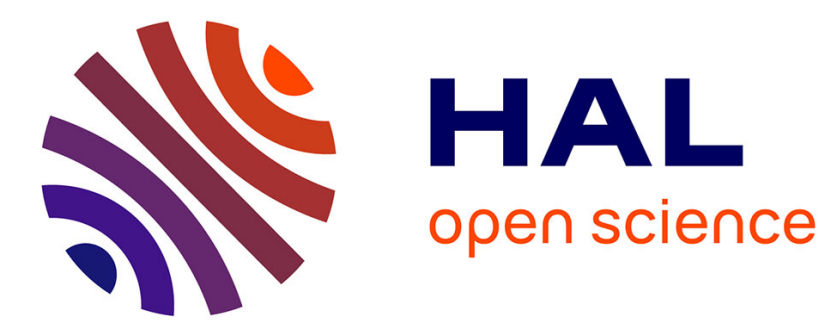

\title{
Enhanced two-photon-absorption using sub-wavelength antennas
}

\author{
B. Vest, B. Portier, F. Pardo, N. Péré-Laperne, E. Steveler, J. Jaeck, C.
} Dupuis, N. Bardou, A. Lemaître, E. Rosencher, et al.

\section{- To cite this version:}

B. Vest, B. Portier, F. Pardo, N. Péré-Laperne, E. Steveler, et al.. Enhanced two-photon-absorption using sub-wavelength antennas. SPIE Photonics West 2015, Feb 2015, SAN FRANCISCO, United States. hal-01121728

\section{HAL Id: hal-01121728 \\ https://hal.science/hal-01121728}

Submitted on 2 Mar 2015

HAL is a multi-disciplinary open access archive for the deposit and dissemination of scientific research documents, whether they are published or not. The documents may come from teaching and research institutions in France or abroad, or from public or private research centers.
L'archive ouverte pluridisciplinaire HAL, est destinée au dépôt et à la diffusion de documents scientifiques de niveau recherche, publiés ou non, émanant des établissements d'enseignement et de recherche français ou étrangers, des laboratoires publics ou privés. 


\title{
Enhanced two-photon-absorption using sub-wavelength antennas
}

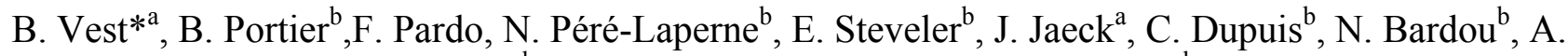

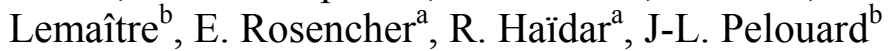 \\ ${ }^{a}$ ONERA The French Aerospace Lab, Chemin de la Hunière, F-91760 Palaiseau, France; \\ ${ }^{b}$ Laboratoire de Photonique et de Nanostructures (LPN-CNRS), Route de Nozay, 91460 Marcoussis, \\ France
}

\begin{abstract}
Degenerate two-photon absorption (TPA) is investigated in a $186 \mathrm{~nm}$ thick gallium arsenide (GaAs) p-i-n diode embedded in a resonant metallic nanostructure. The full device consists in the GaAs layer, a gold subwavelength grating on the illuminated side, and a gold mirror on the opposite side. For TM-polarized light, the structure exhibits a resonance close to $1.47 \mu \mathrm{m}$, with a confined electric field in the intrinsic region, far from the metallic interfaces. A 109 times increase in photocurrent compared to a non-resonant device is obtained experimentally, while numerical simulations suggest that both gain in TPA-photocurrent and angular dependence can be further improved. For optimized grating parameters, a maximum gain of 241 is demonstrated numerically and over incidence angle range of $\left(-30^{\circ} ;+30^{\circ}\right)$. This structure paves the way towards low-noise infrared detection, using non-degenerate TPA, involving two photons of vastly different energies in the same process of absorption in a large bandgap semiconductor material.
\end{abstract}

Keywords: infrared, two photon absorption, nanostructure, photodiode, subwavelength, plasmonics, confinement

\section{INTRODUCTION}

Two-photon absorption (TPA) in semiconductors has drawn much interest in the past 20 years, as it allows photocurrent generation by absorption of subbandgap photons pairs. At present, TPA-based photodetection is commonly used for autocorrelation measurements of pulsed laser sources [1,2] and is under study for other applications such as high-speed signal processing, [3,4] coherent control, [5] and low noise infrared detection. [6-8] Such applications are currently limited by the low efficiency of TPA processes in conventional photodiodes (PDs). To obtain measurable levels of photocurrent, one must either introduce electronic gain (photomultipliers and avalanche photodiodes), [9,10] or increase the light/matter interaction inside the PD. For the latter, a first approach is to integrate the active region in a waveguide structure, $[11,12]$ thus enabling both long interaction lengths and better light confinement. A second approach is to couple the active region to a resonant structure, since TPA increases linearly with the squared light intensity. Enhancements of TPA photocurrent by several orders of magnitude have been demonstrated with Bragg microcavities [13] or photonic cristals. [14] Other resonant structures, such as metallic nanostructures, might also be suitable candidates for improved TPA-based PDs. For example, multiple resonant effects (plasmonic, Fabry-Perot (FP) or guidemode resonances) can be obtained by introducing metallic nanoantenna in thin photodiodes, [15] inducing field enhancements in the active region.

We have investigated the enhancement of TPA in a photodetecting device in which the metallic electrodes play the role of resonant antenna. The device consists in a thin gallium arsenide $\mathrm{p}-\mathrm{i}-\mathrm{n}$ junction, a back-reflecting gold mirror, and a lamellar grating on the illuminated side (see Fig. 1(a) ). For TM-polarized light, the structure exhibits a resonance around $1.47 \mu \mathrm{m}$, with a strong field enhancement in the intrinsic region of the junction. We emphasize that the field is thus localized far from the interfaces, which effectively reduces the chances of having single-photon absorption (SPA) due to defect-assisted transitions at the interfaces, [16] while ensuring a high extraction rate of the free carriers. We demonstrate both numerically and experimentally that the TPA-photocurrent can be enhanced by at least two orders of magnitude at resonance, compared to a non-resonant PD. We also show how TPA gain and angular range can be improved by further optimizing the lateral dimensions of the metal grating. 


\section{DESIGN \& FABRICATION}

The metallic nanostructure was numerically designed using the B-Spline modal method. [17] The permittivity values used for calculations were interpolated from experimental data for gallium arsenide, [18] and calculated using a DrudeLorentz model for gold. [19] TPA was not taken into account for the electric field computations, having negligible effects. The structure parameters, detailed in Fig. 1(a), are $\mathrm{t}=100 \mathrm{~nm}, \mathrm{~L}=186 \mathrm{~nm}, \mathrm{p}=650 \mathrm{~nm}$, and $\mathrm{w}=390 \mathrm{~nm}$. The nominal thicknesses of the p-i-n junction are $50 \mathrm{~nm}$ for the p-layer, $60 \mathrm{~nm}$ for the i-layer, and $76 \mathrm{~nm}$ for the $\mathrm{n}$-layer. For a TM-polarized wave under normal incidence, this structure exhibits a FP resonance at $\lambda=1.486 \mu \mathrm{m}$. The normalized squared intensity (see Fig. 1(c), left) is characterized by a maximum around the middle of the p-i-n junction, confined below the metal stripes, with an increase of nearly three orders of magnitude compared to the incident light intensity I20 . The lateral confinement is quite unusual in the case of a FP resonance. The computation shows that it is due to interferences between the resonant fundamental mode and the \pm 1 diffracted orders generated by the grating. The squared light intensity in the GaAs layer, averaged along $\mathrm{x}$, is shown in Figure 1(c) (right). It is normalized by the squared incident light intensity I20, which incidentally is also the squared intensity in a thick GaAs layer with an antireflection (AR) coating. The minimum value of $\frac{I_{x}^{2}}{I_{0}^{2}}$ is 25 , and the maximum value is 264 . A significant increase of the TPA rate is therefore expected at resonance compared to a non-resonant structure.
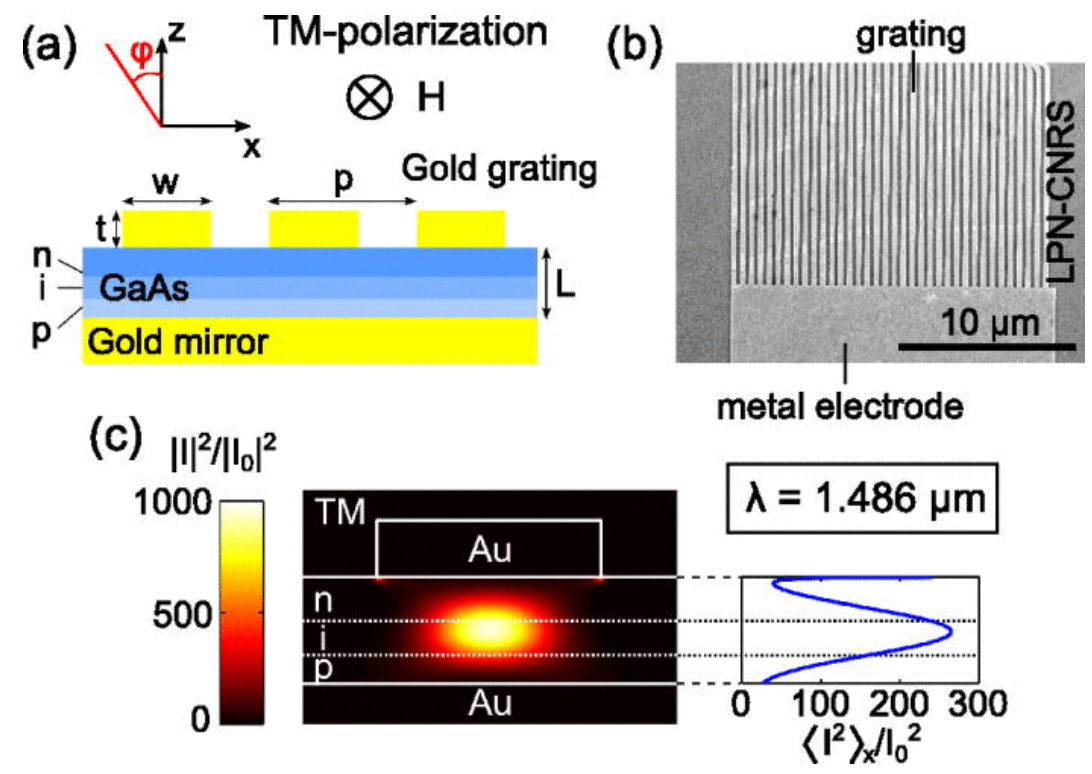

Figure 1 : (a) Schematic of the photodetecting device, consisting in a thin gallium arsenide p-i-n junction of thickness $\mathrm{L}=186 \mathrm{~nm}$, a gold back-reflector, and a top gold lamellar grating of period $\mathrm{p}=650 \mathrm{~nm}$, width $\mathrm{w}=390 \mathrm{~nm}$, and thickness $\mathrm{t}$ $=100 \mathrm{~nm}$. The thicknesses of the GaAs layers are $50 \mathrm{~nm}$ for the p-layer, $60 \mathrm{~nm}$ for the i-layer, and $76 \mathrm{~nm}$ for the $\mathrm{n}$-layer. (b) Scanning electron microscope image (top view) of a $20 \mu \mathrm{m}$ large 1D gold grating and electrode on the GaAs junction. (c) Left: normalized electric field intensity map at $\lambda=1.486 \mu \mathrm{m}$, for a TM-polarized incident wave at normal incidence; right: average field intensity along a period.

Devices were fabricated by an epitaxial layer transfer process, followed by a lift-off technique for the top metallic grating deposition. The $186 \mathrm{~nm}$ p-i-n junction was grown by molecular beam epitaxy on a GaAs substrate with a AlGaAs etch stop layer. $\mathrm{Au} / \mathrm{Ti} / \mathrm{Al}(200 / 20 / 200 \mathrm{~nm})$ layers were deposited on the junction by electron-beam assisted evaporation. The sample was then transferred on Pyrex glass by anodic bonding at $210^{\circ} \mathrm{C}$, and the substrate and etch stop layer were successively removed by selective wet etching. The $20 \mu \mathrm{m} \times 20 \mu \mathrm{m}$ grating patterns were defined by e-beam lithography in a $300 \mathrm{~nm}$ thick layer of poly(methylmethacrylate). After resist development, a $100 \mathrm{~nm}$ Au layer was 
deposited, and lifted off in trichloroethylene. Mesas of size $80 \mu \mathrm{m} \times 30 \mu \mathrm{m}$ were chemically etched around the devices. Eventually, top metallic contacts were made on either side of the gratings by a lift-off technique with optical lithography.

\section{EXPERIMENTAL SETUP AND CHARACTERIZATION}

In the experimental setup, the infrared light source is a nanosecond optical parametric oscillator (OPO) APE nanoLevante that delivers $2 \mathrm{~ns}$ pulses at $20 \mathrm{kHz}$, and can be tuned in the $[1.43 \mu \mathrm{m} ; 1.9 \mu \mathrm{m}]$ range. The output of the $\mathrm{OPO}$ is injected in a monomode fiber. The light is then collimated and linearly polarized. The incident power and orientation of the linear polarization can be controlled with a set of wave plates and a Polarizing Beam Splitter (PBS). The collimated beam is finally focused on the sample with a NA $=0.5$ aspheric lens which allows us to reach quasidiffraction limited beam. The spot size (waist $\mathrm{w} 0=4 \mu \mathrm{m}$, as measured by the knife-edge technique) is smaller than the device under characterization. The half-divergence angle of the focused beam is $8^{\circ}$. The incident power is monitored with a Thorlabs DET10D InGaAs Photodiode, which collects the light reflected by the PBS. Under illumination, the photocurrent is collected and measured by a Keithley 6430 source-meter. A $-0.1 \mathrm{~V}$ reversed bias is applied to the p-i-n diode, which exhibits a dark current of $1.25 \mu \mathrm{A} \mathrm{cm}-2$. Figures 2 (a) $-2(\mathrm{~b})$ show, respectively, the incident power delivered by the OPO source ( P 0 ) and the measured photocurrent ( $\mathrm{J} \mathrm{ph}$ ) for wavelengths in the range $[1.43 \mu \mathrm{m} ; 1.6$ $\mu \mathrm{m}]$. While the incident power gradually increases on the full spectral range, the photocurrent increases rapidly until $\lambda=1.468 \mu \mathrm{m}$ where it is maximum, then decreases. The maximum photocurrent corresponds to the FP resonance predicted at $1.486 \mu \mathrm{m}$. The spectral shift is attributed to the etching of a few $\mathrm{nm}$ of the GaAs n-layer during chemical processes such as selective wet etching and deoxidation. The photocurrent generation process close to resonance is determined by current vs incident power characterization (Fig. 2(c)).

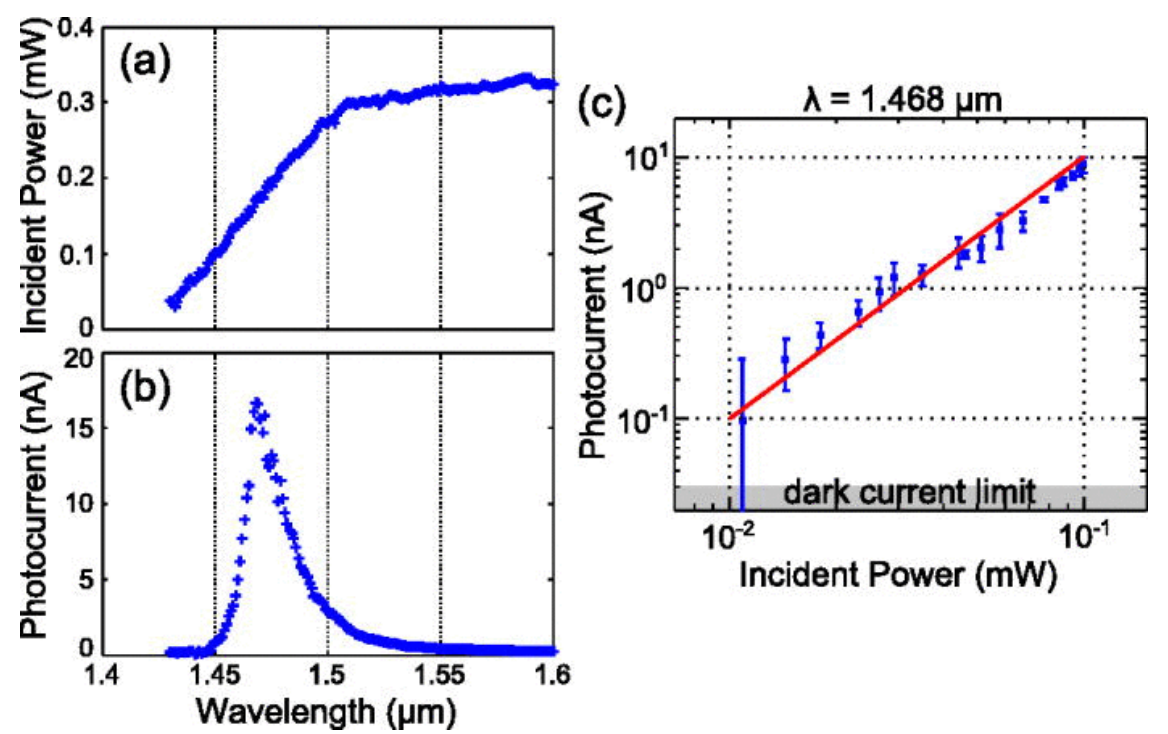

Figure 2 : (a) Incident power delivered by the OPO source vs wavelength. (b) Measured photocurrent vs wavelength. A significant increase appears around $\lambda \sim 1.468 \mu \mathrm{m}$ due to the FP resonance. (c) Photocurrent vs incident power in log scale close to resonance. The quadratic dependence (evidenced by the red line) is typical of photocurrent generation by TPA.

The values show a clear quadratic dependence with the incident optical power, highlighting a TPA mechanism in the photodiode for incident powers in the $[0.01 \mathrm{~mW} ; 0.1 \mathrm{~mW}]$ range. For lower input powers (i.e., below $0.01 \mathrm{~mW}$ ), we were not able to determine the photocurrent generation mechanism (SPA or TPA) because the measured photocurrent was of the same order as the dark current ( $\mathrm{J}$ obs $=30 \mathrm{pA}$ ), with fluctuations $2-3$ times the value of $\mathrm{J}$ obs. These 
observations tend to prove that our design is efficient in promoting TPA over defect-assisted SPA, as the electromagnetic field is enhanced far from the interfaces, where most of the defects are located.

\section{TWO-PHOTON ABSORPTION GAIN FACTOR}

The photocurrent enhancement at resonance is now assessed. In the pure degenerate TPA regime $\left(\frac{E_{g}}{2} \leq \hbar \omega \leq E_{g}\right)$ the intensity which is absorbed by the semiconductor when light propagates along $\mathrm{z}$ is

$$
\frac{d I}{d z}=-\beta_{b u l k} I^{2}
$$

where I is the light intensity and $\beta$ bulk is the TPA coefficient of the bulk semiconductor. TPA absorption over a period of the grating is therefore proportional to the average squared intensity inside the GaAs layer $I_{G a A s}^{2}$. It can be expressed as

$$
I_{A b s}=\beta_{b u l k} G_{T P A} I_{0}^{2} L
$$

where $I_{0}=\frac{P_{0}}{S}$ is the incident light intensity $\left(S=\pi w_{0}{ }^{2}\right)$ and $G_{T P A}=\frac{I_{G a A s}^{2}}{I_{0}^{2}}$ is the TPA gain factor. Considering that an electron-hole pair is created for each TPA event, the total photocurrent generated by the device is

$$
J_{p h}=\frac{e \lambda}{2 h c} \eta_{\text {int }} \beta_{e f f} I_{0}^{2} L S
$$

where $\eta_{\text {int }}$ is the free carrier extraction rate and $\beta_{\text {eff }}=G_{T P A} \beta_{b u l k}$ is an efficient TPA coefficient. For a GaAs photodiode with an AR coating, $G_{T P A}=1$ and $\beta_{\text {eff }}=\beta_{b u l k}=9.6 \mathrm{~cm} . G W^{-1}$ at $1.468 \mu \mathrm{m}$. Assuming an extraction rate of 1 for the fabricated device, we find $\beta_{\text {eff }}=1025 \pm 128 \mathrm{~cm} . G W^{-1} \beta \mathrm{eff}=1050 \pm 128 \mathrm{~cm} \mathrm{GW}-1$ at resonance, i.e., a gain factor of $109 \pm 13$.

The TPA gain factor was also evaluated numerically by calculating the normalized average light intensity $\left\langle\frac{I_{\text {GaAs }}^{2}}{I_{0}^{2}}\right\rangle$ in the GaAs layer between $1.4 \mu \mathrm{m}$ and $1.6 \mu \mathrm{m}$ (see Fig. 3 ). For the same dimensions as the experimental device (blue curve), the gain factor is less than 1 in the off-resonance case and reaches 139 at $\lambda=1.486 \mu \mathrm{m}$. The small discrepancy with the experimental value (109) is attributed to an overestimated free carrier extraction rate. 


\section{OPTIMIZED GRATING PARAMETERS}

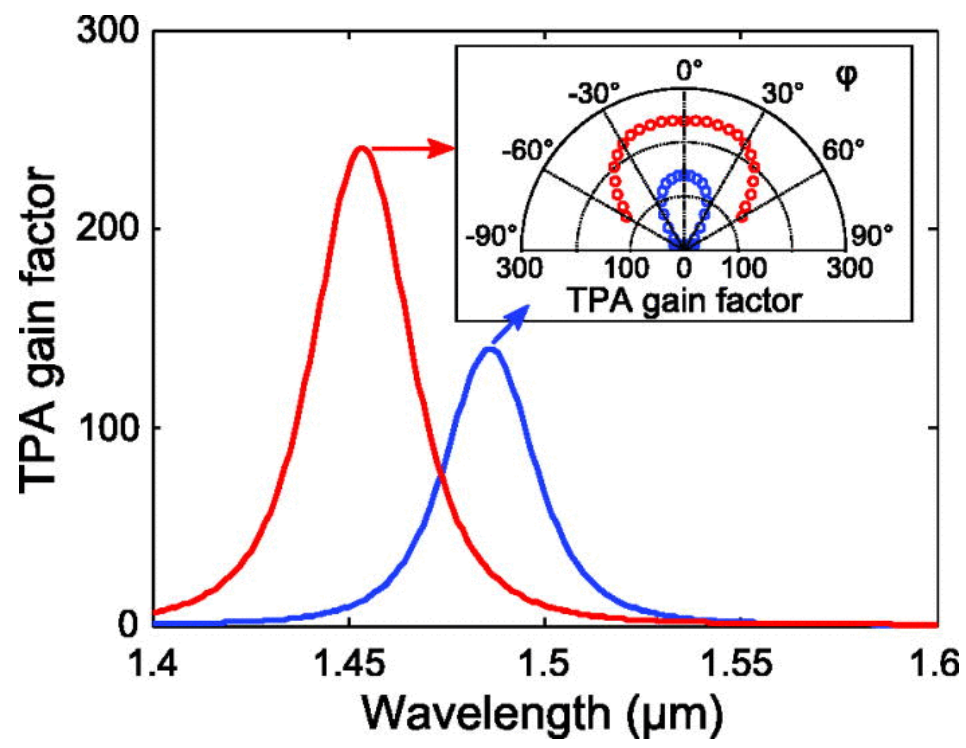

Figure 3 : Calculated TPA gain factor vs wavelength for a TM-polarized wave at normal incidence for two sets of gratings parameters. Blue: $\mathrm{p}=650 \mathrm{~nm}$ and $\mathrm{w}=390 \mathrm{~nm}$, like the fabricated device. Red: $\mathrm{p}=550 \mathrm{~nm}$ and $\mathrm{w}=330 \mathrm{~nm}$ after gain optimization. Inset: gain factor vs incidence angle $\varphi$ (see Fig. 1 ) at $\lambda=1.486 \mu \mathrm{m}$ for the fabricated grating (blue) and $\lambda=1.453 \mu \mathrm{m}$ for the optimized grating (red).

Note that a simple GaAs/gold bilayer in air also induces FP resonances with increased TPA. Assuming a bilayer with a FP resonance at $1.486 \mu \mathrm{m}$, the estimated TPA gain factor at resonance, given by the formula $\frac{3 n_{s}^{2} f^{4}}{8}$ in [20] , is 54. The top metallic grating therefore increases TPA-generated photocurrent by a factor of 2.6 in TM-polarization. Given the technological constraints relative to the grating fabrication, the increment is small, and calls for further improvement of the nanostructure design. As an example, the red curve in Figure 3(a) was obtained by changing the grating parameters to $\mathrm{p}=550 \mathrm{~nm}$ and $\mathrm{w}=330 \mathrm{~nm}$. A maximum gain of $G_{T P A}^{M a x}=241$ is then reached at $\lambda=1.453 \mu \mathrm{m}$, resulting in a 4.4 increase of TPA gain compared to the bilayer. Also, the angular range of the resonance is significantly improved. The inset of Figure 3 shows the gain factor for both the fabricated and optimized gratings at resonance, for several values of the incidence angle $\varphi$ in the (xy)-plane. While the gain associated to the fabricated device at $1.486 \mu \mathrm{m}$ decreases rapidly when $\varphi$ increases, it remains stable for the optimized device at $1.453 \mu \mathrm{m}$, with $\frac{G_{T P A}}{G_{T P A}^{M a x}} \geq 95 \%$ in the $\left[-30^{\circ} ;+30^{\circ}\right]$ range. The wide angular range will allow us to reach better concentration of the incident light on the PD by use of higher NA lenses.

\section{CONCLUSION}

In summary, a two photon detecting device comprising a thin GaAs layer and a resonant metallic nanostructure was designed and fabricated. For TM-polarization, the structure exhibits a FP resonance in the 1.45-1.48 $\mu \mathrm{m}$ range. We demonstrate both experimentally and numerically an enhancement of the TPA-generated photocurrent at resonance by two orders of magnitude, due to high energy confinement in the semiconductor region. After further optimization of grating parameters, the TPA gain factor can be almost independent of the incidence angle over a large angular domain $\left(-30^{\circ} ;+30^{\circ}\right)$, which allows the use of such a device with highly focused beams. These results show that metallic 
nanostructures are an interesting alternative to Bragg microcavities, which can provide higher gains but at the cost of weaker angular range. [21] However, it could also be interesting to replace the gold mirror by a highly reflective Bragg mirror on the backside, thus having a hybrid structure with improved TPA gain (due to better reflectivity on the backside) as well as good angular range and spectral tunability thanks to the grating. Given the cost of e-beam lithography, alternative (cheaper) techniques for fabricating this grating should be preferred, e.g., nanoimprint. Further improvements of the design include 2D metallic gratings to suppress the polarization dependance, or dielectric gratings to reduce energy loss in the metal layers.

Finally, enhanced non-degenerate TPA could be achieved in a similar way with multiresonant metallic nanostructures, which paves the way towards low-noise infrared detection. Non-degenerate TPA is based on the absorption of two photons having vastly different energies. The absorption coefficient associated to ND-TPA is dramatically increased when one of the photon energies becomes very small, being then almost resonant to an intraband transition, the other one becoming almost resonant to the one photon linear interband transition [22].

As shown in [23], non-degenerate TPA allows mid-IR detection in wide bandgap materials. However, this process remains a third order non-linear process, meaning an intrinsically low-efficient process. The design of multiresonant metallic nanostructures, based on our architecture, and allowing an interaction between mid IR signal photons and near IR pump photons is expected to provide an interesting non-degenerate TPA gain factor, and new perspectives for IR detection at high operating temperature.

\section{REFERENCES}

[1] Y. Takagi, T. Kobayashi, K. Yoshihara, and S. Imamura, Opt. Lett. 17, 658 (1992).

[2] J. K. Ranka, A. L. Gaeta, A. Baltuska, M. S. Pshenichnikov, and D. A. Wiersma , Opt. Lett. 22, 1344 (1997).

[3] T. Liang, L. Nunes, M. Tsuchiya , K. Abedin , T. Miyazaki , D. V. Thourhout, W. Bogaerts , P. Dumon , R. Baets, and H. Tsang, Opt. Commun. 265, 171 (2006).

[4] Q. Xu and M. Lipson, Opt. Express 15, 924 (2007).

[5] A. Hayat, P. Ginzburg, and M. Orenstein, Opt. Express 17, 21280 (2009).

[6] A. Hayat, P. Ginzburg, and M. Orenstein, Phys. Rev. B 77, 125219 (2008).

[7] F. Boitier, J.-B. Dherbecourt, A. Godard, and E. Rosencher, Appl. Phys. Lett. 94, 081112 (2009).

[8] D. A. Fishman, C. M. Cirloganu, S. Webster, L. A. Padilha, M. Monroe, D. J. Hagan , and E. W. Van Stryland, Nat. Photonics 5, 561 (2011).

[9] K. Kikuchi , Electron. Lett. 34, 123 (1998).

[10] J. M. Roth , T. E. Murphy, and C. Xu , Opt. Lett. 27, 2076 (2002).

[11]H. Tsang, P. Vasilev, I. White, R. Penty, and J. Aitchison, Electron. Lett. 29, 1660 (1993).

[12] T. Liang, H. Tsang, I. E. Day, J. Drake, A. P. Knights , and M. Asghari, Appl. Phys. Lett. 81, 1323 (2002).

[13] H. Folliot, M. Lynch, A. L. Bradley, T. Krug, L. A. Dunbar, J. Hegarty, J. F. Donegan , and L. P. Barry , J. Opt. Soc. Am. B 19, 2396 (2002).

[14] T. Tanabe , H. Sumikura, H. Taniyama , A. Shinya , and M. Notomi , Appl. Phys. Lett. 96, 101103 (2010).

[15] I. Massiot, C. Colin , N. Péré-Laperne , P. Roca i Cabarrocas , C. Sauvan , P. Lalanne , J.-L. Pelouard , and S. Collin, Appl. Phys. Lett. 101, 163901 (2012). 
[16]B. Vest, E. Lucas, J. Jaeck, R. Haidar, and E. Rosencher, Appl. Phys. Lett. 102, 031105 (2013).

[17]P. Bouchon, F. Pardo, R. Haïdar, and J.-L. Pelouard, J. Opt. Soc. Am. A 27, 696 (2010).

[18]E. D. Palik , Handbook of Optical Constants of Solids ( Academic press, 1998), Vol. 3.

[19] A. D. Rakic, A. B. Djurišic, J. M. Elazar, and M. L. Majewski, Appl. Opt. 37, 5271 (1998).

[20]H. Folliot, M. Lynch , A. Bradley , L. Dunbar , J. Hegarty , J. Donegan , L. Barry , J. Roberts , and G. Hill , Appl. Phys. Lett. 80, 1328 (2002).

[21] T. Krug, W.-H. Guo , J. O'Dowd, M. Lynch, A. Bradley, J. Donegan, P. J. Maguire, L. Barry , and H. Folliot, IEEE Photonics Technol. Lett. 18, 433 (2006).

[22] D. Hutchings and E. Stryland, J. Opt. Soc. Am. B 9, 2065-2074 (1992)

[23] D. Fishman, C. Cirloganu, S. Webster, L. Padilha, M. Monroe, D. hagan and E. Van Stryland, Nature Photonics 5, 561-565 (2011) 